$\Phi=$

\title{
Prevalence of chronic kidney diseases and determinants among TDF users of pregnant and lactating women based on eGFR-cg, and MDRD-4 in hospital setting of north east Amhara, Ethiopia
}

\author{
Eyob G. Gebretsadik ${ }^{1}$ *, Azmach H. Gebregiorgis ${ }^{2}$, Mohammed H. Mohammed ${ }^{3}$, Samuel Z. Kidane ${ }^{4}$ \\ ${ }^{1}$ Wollo University, Federal Ministry of Health, Ethiopia \\ ${ }^{2}$ World Health organization, Ethiopia \\ ${ }^{3}$ Wollo University, Ethiopia \\ ${ }^{4}$ Federal Ministry of Health, Ethiopia \\ *Corresponding author E-mail: egebretsadik29@gmail.com
}

\begin{abstract}
In recent days, it is common to see increasing incidence of Fanconi, proximal kidney tubular damage and chronic kidney diseases-CKD among high risk populations that drew the clinicians' attention to monitor closely. Among these risk populations with potential CKD incidence; HIV positive patients who uses TDF as a component of HAART need to be monitored for the incidence of CKD as a toxicity of TDF before initiation and during treatment despite the fact that the current monitoring practice in Ethiopia in most hospitals remain to be poor. Hence this study aimed at measuring the incidence of CKD among high risk segment of HIV positive pregnant and lactating women who uses TDF as part of their HAART treatment. Using a non-proportionate stratified sampling, a total of $111 \mathrm{HIV+ \text {pregnant }}$ and lactating women who are on TDF based HAART treatment were enrolled to measure the incidence of CKD based on NKF K/DOQI Classification. Using the Android application of Medicalc GFR-cg, and MDRD-4; the prevalence of stage-2 CKD was 16.2 \% (60-89 $\mathrm{ml} / \mathrm{min})$ and Stage 5-CKD/Renal Failure who require dialysis were $3.6 \%(\mathrm{CrCl}<15 \mathrm{ml} / \mathrm{min} / 1.72 \mathrm{~m} 2)$ by both method of calculation $\mathrm{CrCl}$ (GFR-cg and MDRD-4). Women who were lactating had a relative risk of 0.918 (95\% CI lies within 0.845-0.998) of acquiring CKD (P= 0.045). The other associated factors were BMI less than 18.5 ( $\mathrm{P}=0.004$ and adjusted OR of 7.82$)$, WHO clinical stage- 1 ( $\mathrm{P}=0.014$, odds ratio of 5.4 and $95 \% \mathrm{CI}$ of 1.24-24.42), baseline CD4 count $>500(\mathrm{P}=0.02)$, and duration on TDF (> 12 months on treatment) and low haematocrit of 30 had a higher risk of falling into Stage 2 CKD with cohort risk estimate of 4.103 (95\% CI of 1.02, 16.54). The risk estimate of WHO stage 2 to acquire stage-4 CKD was 1.087 (95\% CI of $1.002,1.180)$ statistically significant $(\mathrm{P}=0.05$.). The prevalence of stage $2 \mathrm{CKD}$ among pregnant and lactating women by GFR-cg method of calculation was higher than MDRD-4 calculation. In this study, MDRD-4 method underestimated stage 2 CKD. Hence it is worth and highly recommended to use GFR-cg method in the baseline and during treatment monitoring of TDF toxicity to the kidney particularly for diagnosing the early stage of CKD.
\end{abstract}

Keywords: Acute Kidney Injury; Chronic Kidney Disease; Creatinine Clearance; Glomerular Filtration Rate; Modified Renal Diet Diseases; Renal Failure; TDF.

\section{Introduction}

The transmission of HIV from HIV-positive pregnant mother to child (MTCT) occurred at the time of during pregnancy, labour and delivery and after delivery during breastfeeding that accounts about $15-45 \%$ of transmission of HIV from mother to infants without any intervention (WHO 2014).

Nevertheless, with HAART treatment as recommended by WHO option B+ PMTCT guideline (the lifelong HAART for all HIV positive pregnant and lactating mothers while NVP prophylactic syrup); the probability of HIV transmission has been reduced to lower than 0.02 when combining with elective caesarean section (CS).Since June 2013, Ethiopian FMOH adopted the option B+ option guideline and recommends the initiation of preferred TDF based HAART to all HIV positive mothers during pregnancy and lactation irrespective of the clinical WHO stage or CD4 count for life long. (Gobalappa C, Stover J. 2014)

Tenofovir Disoproxil Fumarate (TDF) is used the first-line preferred regimen It has become a key component in combined ART due to its favourable efficacy and safety profile and being effective in treatment-naive and treatment-experienced patients. (Christopher A Fux, Mathew Simcock. 2014).

Though many studies support the overall good safety of TDF with only sporadic cases of kidney disease; renal tubular damage and impaired creatinine clearance have been increasingly being reported in patients with underlying renal disease and other co-morbid such as diabetes. (Pablo L, Pablo B. 2009).

The national Kidney Foundation (NKF K/DOQI Classification) has defined Chronic Kidney Disease (CKD) as either kidney damage (pathological abnormality or markers of damage) or GFR 
$<60 \mathrm{ml} / \mathrm{min} / \mathrm{m} 2$ for 3 months with the stages of CRD based on the GFR were put as:

Table 1: Table showing Chronic Kidney disease classification (NKF K/DOQI Classification

\begin{tabular}{ll}
\hline Stage & MDRD-4,eGFR-cg $(\mathrm{ml} / \mathrm{min} / 1.72 \mathrm{~m} 2)$ \\
\hline 1. (normal or $\downarrow$ GFR) & $>90 \mathrm{ml} / \mathrm{min}$ \\
2. (mild $\downarrow$ GFR) & $60-89 \mathrm{ml} / \mathrm{min}$ \\
3. (moderate $\downarrow$ GFR) & $30-59 \mathrm{ml} / \mathrm{min}$ \\
4. (Sever $\downarrow$ GFR) & $15-29 \mathrm{ml} / \mathrm{min}$ \\
5. Kidney Failure &, $15 \mathrm{ml} / \mathrm{min}$ or dialysis \\
\hline
\end{tabular}

During pregnancy there is a $40-50 \%$ rise in serum creatinine, hence $\mathrm{CrCl}<90 \mathrm{ml} / \mathrm{min}$ considered as reduced GFR unlike for Adult group $\mathrm{CrCl}$ between $60-90 \mathrm{ml} / \mathrm{min}$ as stage 2 mild renal diseases. (Derek C.2012) However, despite WHO and national guideline recommendation for close monitoring of toxicity and substitution by other NRTI agent: the current practice of toxicity monitoring is poor.

The prevalence of chronic kidney disease (CKD) among people infected with HIV reaches about $30 \%$ which increasingly has become a cause for morbidity and mortality. (Primary care of Veterans with HIV. April 2009). However, the reported prevalence of TDF-related kidney injury from randomized clinical trials ranges from $1 \%$ (as confirmed by abnormal serum creatinine $>1.5 \mathrm{mg} / \mathrm{dl}$ and phosphorus $<2.0 \mathrm{mg} / \mathrm{dl}$ ) underestimate than serum creatinine (5\%). There was also high discrepancy with prevalence of CKD (8.4\%7) and total tubular damage of TDF (26\%) and hospitalization rate $(31.4 \%)$ and mortality of $18.2 \%$. (Mohammed I Danjuma, Nurul H Mohammed 2014). Most Studies showed worsening of impaired GFR in patients on TDF based HAART but usually underlying renal dysfunction. (Benjamin Y, Kate B. 2009).

Though toxicity monitoring of TDF for CKD has been recommended for early management as discontinuation of TDF or substituting with other agent ( when $\mathrm{CrCl}<60 \mathrm{ml} / \mathrm{min}$ ) or dose adjustment when there is no option for other preferred regimen; the decline in estimated GFR $(\mathrm{CrCl}<90 \mathrm{ml} / \mathrm{min})$ for pregnant and lactating women has been underestimated owing to the rise of 40$50 \%$ serum creatinine during pregnancy state .So far hypertension, as duration on HAART, and TDF exposure remained significantly associated to CKD ( $15 \%$ increased prevalence of CKD per each year.) (Andreia M, Jorge T Jr. 2012)

The other factors associated were both underweight and low $\mathrm{Hgb}$ level (Anemia) were most strongly associated factors in spite of the egg and chicken dilemma which one of them has brought it which. According to Ethiopian Demographic Health Survey (EDHS) 2011 data, 17\% of pregnant women in Ethiopia were anemic while $27 \%$ of pregnant were underweight which substantially hamper the incidence among HIV+ positive of pregnant and lactating. Similarly, relevant to the underweight factors such as BMI; the bone density mass in HIV positive patients who uses TDF based HAART were often low which has been related as well with co-morbidity of CKD.

Having reviewing all those factors associated with reduced $\mathrm{CrCl}$; pregnancy and its medical complication as a single entity often falls into high risk category for developing chronic renal diseases. In general, hypertensive disease of pregnancy ranks among the two top causes of maternal mortality irrespective of high HIV comorbidity aside the medical complication of renal and cardiac disease during pregnancy. (EDHS 2011).

\section{Methods and result}

This hospital based cross sectional study design which is both descriptive and analytic aimed at measuring the prevalence of CKD among TDF users of HIV+ pregnant and lactating women and its associated factors and determinants through measuring serum Creatinine and calculating the $\mathrm{CrCl}$ by simple android based mobile application (both a GFR-cg, and MDRD-4). Factors and determinants associated with $\mathrm{CKD}$ and impaired $\mathrm{CrCl}$ were analyzed through logistic regression for adjusted odds ratio among study subjects of pregnant and lactating mothers who were on TDF based HAART. The study was conducted in north-eastern part of Ethiopia in the region of Amhara within public hospital settings of selected 3 Hospitals from 3 zones namely Dessie referral, Woldiya and Tefera Hailu memorial Hospital. The study period was 1 st November 2014 to 31st of June 2015.

Corrected sample size of HIV+ pregnant and lactating women $(\mathrm{n}=102)$ were selected using a systematic randomly sampling method allocated proportional sample size for 3 stratified zones among women enrolled n PMTCT program enrolled from June 2013 to June 2015.

Health care professionals at the level of Health officers and Physicians were recruited as data collectors and trained practically on 20 study subjects under direct supervision.

Measurement of weight and height were taken by data collectors after interview with other clinical examination. Serum creatinine, fasting blood sugar and hematocrit were measured in the next day after the interview in the hospital laboratory which was accredited as 3 star by national assurance body for its quality.

Data inputs from the questionnaire were entered into android application of Medicalc 8.4 for calculation of GFR-cg, MDRD-4 and GFR-EPI. Target Idea body weight (IBW) and BMI were also calculated with its embedded application output which then classify into standard BMI classification (high, Normal, and low). The android application automatically rejects when abnormal variable and values entered with capability of reducing error made during manual calculation.

Independent variables as: weight, BMI, Hematocrit, Baseline CD4, and WHO clinical stage, type of regimen of First line, duration of HAART, duration of TDF based HAART, status of blood pressure (Hypertension (systolic $>160 \mathrm{mmHg}$, and diastolic $>90$ $\mathrm{mmHg}$ ) and status Diabetic Mellitus (Fasting serum glucose 127 $\mathrm{mg} / \mathrm{dl}$ ) were assessed.

Data were entered into epi info for cleansing and exported to SPSS version 22 for analysis of multivariate and binary logistic regression for computing adjusted odds ratio, relative risk and cohort risk within $95 \% \mathrm{CI}$ and statistical significance (P-value $<0.05)$.

Hence the outcome abnormal Creatinine Clearance $(\mathrm{CrCl})$ measured as;

- Glomerular Filtration rate by Cockcroft Gault (GFR-cg) formula (as 3 inputs; age, weight and serum creatinine) and multiplied by 0.85 for women. $(90-131 \mathrm{ml} / \mathrm{min})=$ $(140$-age $) *$ Weight $/ 72 * \mathrm{Cr}$ (Female value $=$ multiple result by 0.85$)$.

- Modified diet renal disease (MDRD-4) formula (4 inputs, simplified with age, gender, ethnic factor and serum creatinine) also Levey equation and (90-131 $\mathrm{ml} / \mathrm{min})=(186.3 * \mathrm{Cr}-1.154 *$ age -0.203 GN F* ET F $)$.

GFR was said to be normal when calculated $\mathrm{CrCl} 90-131 \mathrm{ml} / \mathrm{min} /$ per $1.73 \mathrm{~m}^{2}$. CKD was defined as estimated Glomerular filtration rate (cGFR) creatinine clearance (estimated by Cockcroft-Gault equation) $<60 \mathrm{ml} / \mathrm{min}$ per $1.73 \mathrm{~m}^{2}$ in the absence pregnancy and lactation while reduced when the GFR between $60-89 \mathrm{ml} / \mathrm{min}$ or presence of proteinuria with normal cGFR. 


\section{Result}

A total of 111 study subjects were enrolled in the study larger than the pre-determined sample size $(n=102) .55 \%(n=61)$ of enrolled study subjects were lactating while $45 \%(\mathrm{n}=50)$ were pregnant. Age, BMI, weight and height has a normally distributed of all the study subjects. The age of subjects has a normal distribution with mean of 28.9 years and SD of 4.7. The mean and standard deviation of weights of subjects were $56.9 \mathrm{Kg}$ and 10.8 respectively The mean and standard deviation of heights of subjects were 158.7 and 6.2 respectively.

Of all the study subjects, $6.3 \%$ of them had a SBP measured above $130 \mathrm{mmHg}$ while $1.9 \%$ of study subjects have a diastolic $\mathrm{BP}$ of above $90 \mathrm{mmHg}$.

With respect to the clinical stage at baseline for initiation of TDF based HAART; it was $57.7 \%(n=64)$ initiated at WHO stage-2 while $7.2 \%(\mathrm{n}=8)$ of them initiated at WHO stage- 1 . However, the TDF bases HAART initiation profile based on the immunological criteria as CD4 measure showed that; $27 \%$ of clients were initiated on TDF based HAART at CD4 count $>500$ while $27 \%$ at less than 200

\subsection{Prevalence of stages of CKD by MDRD-4}

For the Serum Creatinine was measured for $86.5 \%$ ( $\mathrm{n}=96)$ study subjects; the denominator for the prevalence of different stages of CKD remained to be $96 \mathrm{HIV}+$ pregnant and lactating clients (94.1 $\%$ of the sample determined).

Of all 96 study subjects, $82.9 \%$ (n=92) study subjects had normal range of creatinine clearance $(90-131 \mathrm{~mm} / \mathrm{min})$ and $13.5 \%$ had high abnormal GFR $(\mathrm{CrCl}>131 \mathrm{ml} / \mathrm{min})$ while $3.6 \%(\mathrm{n}=4)$ of study subjects had stage-5 Chronic Kidney disease (CKD).

\subsection{Prevalence of CKD using eGFR-cg}

Unlike the MDRD-4 estimates for the normal range of $\mathrm{CrCl}(82.9$ $\%)$; the eGFR-method estimated $24.3 \%(\mathrm{n}=27)$ of study subjects had $\mathrm{CrCl}$ of normal level (stage-1: $90-131 \mathrm{ml} / \mathrm{min}$ ). About $42.3 \%$ $(n=47)$ of the study subjects have high abnormal GFR of greater than $131 \mathrm{ml} / \mathrm{min} / 1.72 \mathrm{~m}^{2}$.

However, $16.2 \%(\mathrm{n}=18)$ have reduced stage 2 mild (60-89 $\mathrm{ml} / \mathrm{min}$ ) renal disease while $3.6 \%(\mathrm{n}=4)$ of them had stage 5 (less than $15 \mathrm{ml} / \mathrm{min} / 1.72 \mathrm{~m} 2$ ) kidney failure which requires hospitalization for dialysis.

\section{Analysis of factors and determinants}

Among factors and determinants computed, BMI <18.5 was associated with stage- $2 \mathrm{CKD}(\mathrm{P}=0.004)$ with odds ratio $=5.29$ with 95\% CI $(1.56,18.01)$. The association of lower BMI less than 18.5 with stage 4 CKD not statistically significant $(\mathrm{P}=0.419)$.

The initiation of TDF base HAART at baseline of WHO stage-1 was significantly associated with stage- $2 \mathrm{CKD}$ with odds ratio of 5.147 and the $95 \%$ CI lies within $(1.25,21.24)(\mathrm{P}=0.014)$ while weak cohort risk estimate (RR) 0.352 and the $95 \%$ CI value lie within $(0.171,0.725)$.

The association between baseline WHO stage 2 and CKD was not significant $(\mathrm{P}=0.05)$ with risk estimated to be 1.087 with $95 \% \mathrm{CI}$ lies within $(1.002,1.180)$. Baseline CD4 count at initiation of HAART > 500 was significantly associated with stage-2 CKD $(\mathrm{P}=0.02)$ with cohort risk estimate of 4.103 with $95 \% \mathrm{CI}$ lies with a range of $(1.02,16.54)$.
The other association factors along mentioned earlier was duration of TDF based HAART (duration > 12 months) with stage-2 CKD was not significant $(\mathrm{P}=0.66)$ despite the fact that $3.6 \%(\mathrm{n}=4)$ of women who were not lactating (Pregnant) developed stage-5 CKD (less than $15 \mathrm{ml} / \mathrm{min} / 1.72 \mathrm{~m} 2)$ were significant $(\mathrm{P}=0.045)$ with relative risk (RR) of 0.918 and $95 \%$ CI of $0.845-0.998$.

Table 2: Factors That Determine the Prevalence of Stage 2 Mild Form of Renal Disease and AOR

\begin{tabular}{lllll}
\hline \multicolumn{5}{l}{ Stage 2 mild renal disease (less than $89 \mathrm{ml} / \mathrm{min} / 1.71 \mathrm{~m} 2)$} \\
BMI & $<18.8$ & P-Value & OR & $95 \%$ CI \\
Baseline WHO & Stage 1 & 0.004 & 5.29 & $(1.56,18.01)$ \\
Baseline CD4 & $>500$ & 0.02 & 5.147 & $(1.25,21.24)$ \\
Mo. on TDF & $<12$ mo. & 0.066 & 4.103 & $(1.02,16.54)$ \\
\hline
\end{tabular}

Table 3: The Relative Risk Estimate for Stage 4 and 5 CKD Stage 4 and more CKD less than $60 \mathrm{ml} / \mathrm{min} / 1.71 \mathrm{~m} 2$ )

\begin{tabular}{lllll}
\multicolumn{5}{l}{ Stage 4 and more CKD less than $60 \mathrm{ml} / \mathrm{min} / 1.71 \mathrm{~m} 2)$} \\
Baseline WHO & Stage 2 & P-Value & RR & $95 \%$ CI \\
Lactating & No & 0.05 & 1.087 & $(1.002,1.18)$ \\
\hline
\end{tabular}

\section{Discussion}

The prevalence of stage-5 CKD among HIV+ pregnant and lactating women as measured by the method of MDRD-4 was $3.4 \%$ ( $\mathrm{n}=4$ ) while mild form of kidney disease (stage-2) were not identified by MDRD-4 while GFR-cg have captured $16.2 \%(n=18)$ of stage- 2 . However, $3.6 \%(n=4)$ of the study subjects have stage-5 (less than $15 \mathrm{ml} / \mathrm{min} / 1.72 \mathrm{~m} 2$ ) that require dialysis were not lactating. Hence in a routine monitoring practice for early diagnosis of mild form of stage-2 CKD; it is the eGFR-cg method preferred than MDRD-4.

Nevertheless, the overall prevalence of CKD in this study (19.6 $\%)$ nearly closer to the MHRA study (26\%). The prevalence of stage-5 CKD (renal failure that require dialysis) was $3.4 \%$ higher than $2 \%$ of the Thomas Street Health Center in Houston. (Hana M, Larry T, et al. 2006). Similarly, the overall prevalence of stage-2 in our study was $16.4 \%$ nearly doubled as compared to the $8.8 \%$ stage 2 prevalence of Thomas Street.

A comparison of closer independent characteristics with respect to sex and pregnancy status from Malawi study $(90.8 \%$ of female study participants) had a $6.9 \%$ prevalence of stage- 5 CKD nearly doubled higher than the $3.4 \%$ prevalence of this study. Among determinants, the prevalence of CKD among treatment group whose CD4 count of $>200$ ( 1 year of TDF treatment) in this study was $3.4 \%$ lower than the $8.4 \%$ of the Brazil study. (Andreia M, Jorge T Jr. 2012)

The other factor that determine the prevalence of CKD among the study subjects was lean body mass as measured by MUAC for pregnancy (owing to the expected weight gained from fetal and placental growth) has shown a significant association for underweight was $12.6 \%(\mathrm{P}=0.005$ and $\mathrm{OR}$ of 5.2$)$ which was similar to the Malawi study (OR of 5.4). (Derek C. 2012)

Nevertheless, the use of eGFR less than $50 \mathrm{ml} / \mathrm{min}$ as a cut of for diagnosing CKD by the Malawi study; the significant association of lower CD4 count and higher incidence of CKD has similar association to our study for CD4 count between $200-350 / \mathrm{mm}^{3}$ with odds ratio of 5.2. Moreover, the duration of TDF treatment (more than 12 months) has also significantly associated $(\mathrm{P}=0.018$ and crude odds ratio of 4.38) with Stage 2 decline of GFR which is similar to many studies. (Hana M. et al. 2006, Andreia M. et al 2012, Christopher A Fux et al 2014) 


\section{Conclusion}

The prevalence of stage-2 CKD (as a mild form of renal disease) among TDF based HAART users of pregnant and lactating women were high based on the GFR-cg calculation while the MDRD-4 underestimates stage 2 decline of GFR for early detection.

The prevalence of stage 2 kidney diseases is high among the pregnant and lactating mothers with lower BMI of below18.5, WHO clinical stage of 1 and 2, and higher CD4 count are among the factors that determine the decline of GFR to stage where the duration of TDF based HAART would be significant in larger sample size.

CKD of stage 5 is more associated in those women were not lactating that require further investigation which was beyond the scope of this study. The monitoring of toxicity through GFR-cg is preferred methods than the MDRD-4 for early detection in routing clinical practice.

\section{Acknowledgement}

Our deepest gratitude and warm appreciation goes to those care provider working in the ART and MCH clinic of Dessie, Woldiya, Sekota Tefera Hailu memorial, and Hidar 11 memorial hospital for their relentless effort and consistent support in their tireless data recording and cooperation in the management of patients as to the recommendation and advise.

We would like to extend our deepest appreciation and gratitude to the post-graduate staffs of Wollo university health science college for their invaluable comments and suggestions to the final work of this original research that would provide an important insight to the prevention and early management of chronic kidney diseases that prevail as a result of ARV drug toxicity.

Last but not least, our deep gratitude would be extended to the PEPFAR-CDC, I-TECH Ethiopia, Wollo University, Amhara RHB of South Wollo zone, Tefera Hailu and Hidar 11 Memorial hospital, Woldiya hospital and Dessie hospital for their coordinated lifesaving fee free HAART and PMTCT program with all technical and financial support.

\section{References}

[1] WHO. (2014), HIV/AIDS. Mother-to-child transmission of HIV.

[2] Gobalappa C, Stover J. (2014). The cost benefit of B+ option for the prevention of mother-to- child transmission of HIV/AIDS. Jan; 28.

[3] Ethiopian FMOH. (2013), National B+ Option PMTCT guideline.
[4] Christopher A Fux, Mathew Simcock. (2014), TDF use is associated with reduction in calculated GFR in Swiss HIV cohort. Antiretroviral therapy.

[5] Joel G, Schlomo S. (2004), Efficacy and safety of TDF versus Stavudine combination HAART in treatment naive patients, a 3 year randomized trial. JAMA. 292(2):191-201. https://doi.org/10.1001/jama.292.2.191.

[6] Mohammed I. Danjuma, Nurul H. Mohammed. (2014), an investigation of the pattern of kidney injury in HIV positive person exposed to Tenofovir Disoproxil fumarate: an examination of large population data base (MHRA database). Int. Journal STD AIDS. 24(4):273-279. https://doi.org/10.1177/0956462413504747.

[7] Andreia M, Jorge T Jr. (2012), Prevalence and risk factor associated with to Chronic Kidney disease in HIV infected patients on HAART and undetectable viral load in Brazil... PLoS One.

[8] Derek C. (2012), Tenofovir Use and Renal Insufficiency among Pregnant and General Adult Population of HIV infected, ARTNaive Individuals in Lilongwe. PLoS One -7.

[9] Ethiopian Demographic and Health Survey.2011.

[10] Stefan M, Florian B. (2005), antiretroviral therapy with Tenofovir is associated with mild renal dysfunction. AIDS, 19:93-99. https://doi.org/10.1097/00002030-200501030-00012.

[11] Patricia M. Flynn, Mark M. (2011), Pharmacokinetics and safety of single dose TDF and Emtricitabine in HIV infected pregnant women and their infants. American society for microbiology.

[12] Sergio P, Felix G. (2005), Low frequency renal function impairment during one-year of therapy with TDF containing regimen in real world. AIDS patients care and STDs. July 19(7):421-424.

[13] Benjamin Y, Kate B. (2009) Renal function in patients with preexisting renal disease receiving TDF containing HAART. AIDS patient care and STDs. August; 23(8)

[14] Steven V, Katarina K. (2011), Prevalence of renal impairment among HIV-infected antiretroviral person eligible for HAART in rural Zimbabwe. Medicine sans Frontier.

[15] Pablo L, Pablo B. (2009), Kidney tubular dysfunction in the absence of impaired Glomerular filtration dysfunction. AIDS. 23:689-696. https://doi.org/10.1097/QAD.0b013e3283262a64.

[16] Primary care of Veterans with HIV. (2009). Renal disease: Organ system and metabolic. April 2009, latest updated October 2011.

[17] Gallant, E. (2008), A 3-year renal safety of TDF versus thymidine analogue containing regimen HAART in treatment naive patients. AIDS. October :22(16)

[18] Leigh P, Doug T. (2007), TDF for prevention of HIV infection in Women: A phase 2 double blind randomized placebo control, trial.

[19] Poizot M, Solas C. (2013), renal impairment in patients receiving TDB based combined ART: Impact of TDF trough concentration. J Acquired Immune Deficiency Syndrome.

[20] Santiago M, Pere D, et al. (2006), Renal safety of TDF in HIV-1 treatment experience patients' adverse event related to prior NRTI use: Data from a prospective, observational and multicenter study. J Acquired Immune Deficiency Syndrome. July; 42 (3).

[21] Hana M, Larry T, et al. (2006), Serum creatinine changes in HIV sero-positive patients receiving TDF. AIDS. 20 (5)

[22] K/DOQI Guideline for CKD.

[23] Mark D, Abby G, et al, (2013), Tenofovir associated proteinuria. AIDS.2013; https://doi.org/10.1097/QAD.0b013e32835883bf.
27:479-485. 